\title{
Hadron Production in Hadron-Hadron and Lepton-Hadron Collisions ${ }^{\star}$
}

\author{
Bernd A. Kniehl \\ II. Institut für Theoretische Physik, Universität Hamburg, Luruper \\ Chaussee 149, 22761 Hamburg, Germany
}

\begin{abstract}
Summary. We review a recent global analysis of inclusive single-charged-hadron production in high-energy colliding-beam experiments, which is performed at nextto-leading order (NLO) in the parton model of quantum chromodynamics endowed with nonperturbative fragmentation functions (FFs). Comparisons of $p \bar{p}$ data from CERN Sp $\bar{p}$ S and the Fermilab Tevatron and $\gamma p$ data from DESY HERA with the corresponding NLO predictions allow for quantitative tests of the scaling violations in the FFs and their universality. We emphasize the potential of new measurements at the Tevatron to place tight constraints in the large- $x$ region and on the gluon $\mathrm{FF}$, complementary to those from $e^{+} e^{-}$data, which are indispensible in order to reliably predict the $\pi^{0}$ background for the $H \rightarrow \gamma \gamma$ signal of the intermediate-mass Higgs boson at the Tevatron and the CERN LHC. Adopting a similar theoretical framework for $b$-hadron production, we show that the notorious excess of the Tevatron data over existing theoretical calculations can be ascribed, at sufficiently large values of $p_{T}$, to nonperturbative fragmentation effects inadequately included previously.
\end{abstract}

\section{Introduction}

In the framework of the QCD-improved parton model, the inclusive production of single hadrons is described by means of fragmentation functions (FFs) $D_{a}^{h}\left(x, \mu^{2}\right)$. The value of $D_{a}^{h}\left(x, \mu^{2}\right)$ corresponds to the probability for the parton $a$ produced at short distance $1 / \mu$ to form a jet that includes the hadron $h$ carrying the fraction $x$ of the longitudinal momentum of $a$. Unfortunately, it is not yet possible to calculate the FFs from first principles, in particular for hadrons with masses smaller than or comparable to the asymptotic scale parameter $\Lambda$. However, given their $x$ dependence at some energy scale $\mu$, the evolution with $\mu$ may be computed perturbatively in QCD using the timelike Altarelli-Parisi (AP) equations [1]. This allows us to test QCD quantitatively within one experiment observing single hadrons at different values of centreof-mass $(\mathrm{CM})$ energy $\sqrt{s}$ (in the case of $e^{+} e^{-}$annihilation) or transverse momentum $p_{T}$ (in the case of scattering). Moreover, the factorization theorem guarantees that the $D_{a}^{h}\left(x, \mu^{2}\right)$ functions are independent of the process in which they have been determined and represent a universal property of $h$.

\footnotetext{
* To appear in the Proceedings of the 14th Topical Conference on Hadron Collider Physics (HCP 2002), 29 September - 4 October 2002, Karlsruhe, Germany.
} 
This enables us to make quantitative predictions for other types of experiments as well.

During the last seven years, the experiments at CERN LEP1 and SLAC SLC have provided us with a wealth of high-precision information on how partons fragment into low-mass charged hadrons $\left(h^{ \pm}\right)$. The data partly comes as light-, $c$-, and $b$-quark-enriched samples without [2,3] or with identified final-state hadrons $\left(\pi^{ \pm}, K^{ \pm}\right.$, and $\left.p / \bar{p}\right)$ [ 四 or as gluon-tagged three-jet samples without hadron identification [5.6. 6 . Motivated by this new situation, the author, together with Kramer and Pötter, recently updated, refined, and extended a previous analysis 7 by generating new leading-order (LO) and next-to-leading-order (NLO) sets of $\pi^{ \pm}, K^{ \pm}$, and $p / \bar{p}$ FFs [8]. By also including in our fits $\pi^{ \pm}, K^{ \pm}$, and $p / \bar{p}$ data (without flavour separation) from PEP [9], with CM energy $\sqrt{s}=29 \mathrm{GeV}$, we obtained a handle on the scaling violations in the FFs, which enabled us to determine the strong-coupling constant $\alpha_{s}^{(5)}\left(M_{Z}\right)$.

The formation of $D$ and $B$ mesons from $c$ and $b$ quarks, respectively, is a genuinely nonperturbative process, so that it is indispensable to employ nonperturbative FFs. Furthermore, if the characteristic energy scale $\mu$ is large against the heavy-quark mass $m_{c}\left(m_{b}\right)$, then the QCD-improved parton model with $n_{f}=4\left(n_{f}=5\right)$ active quark flavours is the appropriate theoretical framework, which allows for coherent analyses of data from $e^{+} e^{-}$, lepton-hadron, and hadron-hadron collisions 10,11.

This contribution is organized as follows. In Sect. 2, we present some details of our global fits 8 and assess the quality of the resulting FFs. We also discuss the determination of $\alpha_{s}^{(5)}\left(M_{Z}\right)$ from the scaling violations in the FFs [12]. In Sect. 3, we present comparisons of our NLO predictions for inclusive charged-hadron production [13] with $p \bar{p}$ data from $\mathrm{S} p \bar{p} \mathrm{~S}$ [14] and the Tevatron $[15$ and with $\gamma p$ data from HERA $[16]$. In Sect. A, we demonstrate that the $p_{T}$ distribution of inclusive $B$-meson hadroproduction measured by CDF [17, 18] is well described at NLO in the QCD parton model with $n_{f}=5$ endowed with nonperturbative FFs, once the latter are fitted to LEP1 data [19. Our conclusions are summarized in Sect. 5 .

\section{Determination of the FFs}

The NLO formalism for extracting FFs from measurements of the cross section $d \sigma / d x$ of $e^{+} e^{-} \rightarrow h+X$ is comprehensively described in [7]. We work in the modified minimal-subtraction $(\overline{\mathrm{MS}})$ renormalization and factorization scheme and choose the renormalization and factorization scales to be $\mu_{R}=\mu_{F}=\xi \sqrt{s}$, except for gluon-tagged three-jet events, where we put $\mu_{R}=\mu_{F}=\xi \times 2 E_{\text {jet }}$, with $E_{\text {jet }}$ being the gluon-jet energy in the CM frame. Here, the dimensionless parameter $\xi$ is introduced to determine the theoretical uncertainty in $\alpha_{s}^{(5)}\left(M_{Z}\right)$ from scale variations. As usual, we allow for variations of $\xi$ between $1 / 2$ and 2 about the default value 1 . 
Our strategy was to only include in our fits LEP1 and SLC data with both flavour separation and hadron identification [ [4], gluon-tagged threejet samples with a fixed gluon-jet energy [5], and the $\pi^{ \pm}, K^{ \pm}$, and $p / \bar{p}$ data sets from the pre-LEP1/SLC era with the highest statistics and the finest binning in $x$ [9. The $\chi^{2}$ value per fitted data point turned out to be $\chi_{\mathrm{DF}}^{2}=0.97(0.98)$ at LO (NLO). The goodness of our fit may also be judged from Figs. 1 (a) and (b), where our LO and NLO fit results are compared with the ALEPH, DELPHI, OPAL, and SLD data [4. us for cross checks [2, 3, 6, 20,21. In particular, we probed the scaling violations in the FFs through comparisons with $\pi^{ \pm}, K^{ \pm}$, and $p / \bar{p}$ data from DESY DORIS and PETRA, with CM energies between 5.4 and $34 \mathrm{GeV}$ [20]. Furthermore, we tested the gluon $\mathrm{FF}$, which enters the unpolarized cross section only at NLO, by comparing our predictions for the longitudinal cross section, where it already enters at LO, with available data [2,21]. Finally, we directly compared our gluon FF with the one recently measured by DELPHI in threejet production with gluon identification as a function of $x$ at various energy scales $\mu$ [6]. All these comparisons led to rather encouraging results. We also verified that our FFs satisfy reasonably well the momentum sum rules, which we did not impose as constraints on our fits.

Since we included in our fits high-quality data from two very different energies, namely 29 and $91.2 \mathrm{GeV}$, we were sensitive to the running of $\alpha_{s}(\mu)$ and, therefore, able to extract values of $\Lambda \frac{(5)}{\mathrm{MS}}$. We obtained $\Lambda_{\mathrm{MS}}^{(5)}=88_{-31-23}^{+34} \mathrm{MeV}$ at $\mathrm{LO}$ and $\Lambda_{\mathrm{MS}}^{(5)}=213_{-73_{-29}^{+75}+22} \mathrm{MeV}$ at NLO, where the first errors are experimental and the second ones are theoretical. From the LO and NLO formulas for $\alpha_{s}^{\left(n_{f}\right)}(\mu)$, we thus obtained $\alpha_{s}^{(5)}\left(M_{Z}\right)=0.1181_{-0.0069}^{+0.0058+0.0006}$ (LO) and $\alpha_{s}^{(5)}\left(M_{Z}\right)=0.1170_{-0.0069}^{+0.0055}{ }_{-0.0025}^{+0.0017}$ (NLO), respectively, which is already included in the most recent world average by the Particle Data Group [22]. As expected, the theoretical error on $\alpha_{s}^{(5)}\left(M_{Z}\right)$ is significantly reduced as we pass from LO to NLO. We observe that our LO and NLO values of $\alpha_{s}^{(5)}\left(M_{Z}\right)$ are quite consistent with each other, which indicates that our analysis is perturbatively stable. The fact that the respective values of $\Lambda_{\mathrm{MS}}^{(5)}$ significantly differ is a well-known feature of the $\overline{\mathrm{MS}}$ definition of $\alpha_{s}^{\left(n_{f}\right)}(\mu)$ [23.

\section{Global Analysis of Collider Data}

Recently, we extended our previous tests of scaling violations 8] to higher energy scales by confronting new data of $e^{+} e^{-} \rightarrow h^{ \pm}+X$ from LEP2 [24], with $\sqrt{s}$ ranging from $133 \mathrm{GeV}$ up to $189 \mathrm{GeV}$, with NLO predictions based on our FFs 13. Furthermore, we quantitatively checked the universality of our FFs by making comparisons with essentially all available high-statistics data on inclusive charged-hadron production in colliding-beam experiments [13. This includes $p \bar{p}$ data from the UA1 and UA2 Collaborations [14] at Spp $\mathrm{S}$ and from the CDF Collaboration 15] at the Tevatron, $\gamma p$ data from 


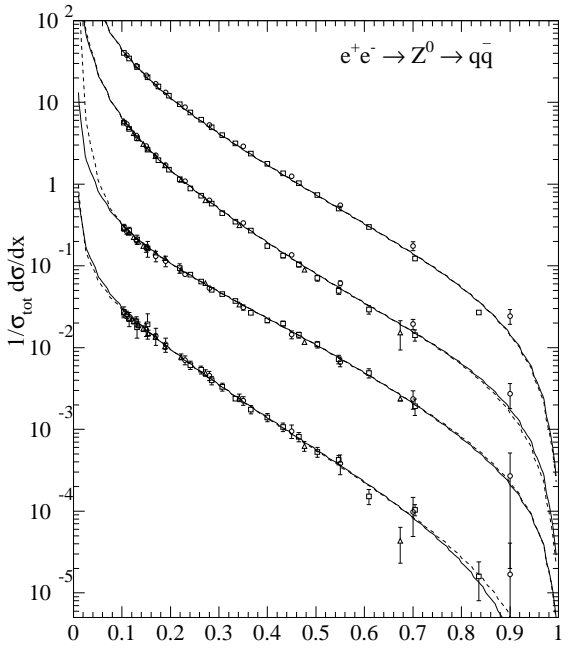

(a)

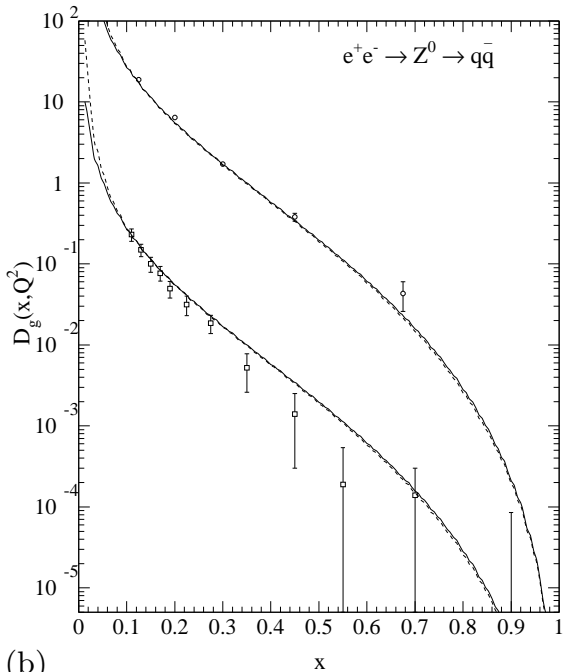

(b)

Fig. 1. (a) Comparison of data on inclusive charged-hadron production at $\sqrt{s}=$ $91.2 \mathrm{GeV}$ from ALEPH (triangles), DELPHI (circles), and SLD (squares) 4 with our LO (dashed lines) and NLO (solid lines) fit results [8]. The upmost, second, third, and lowest curves refer to charged hadrons, $\pi^{ \pm}, K^{ \pm}$, and $p / \bar{p}$, respectively. (b) Comparison of three-jet data on the gluon FF from ALEPH with $E_{\text {jet }}=26.2 \mathrm{GeV}$ (upper curves) and from OPAL with $E_{\text {jet }}=40.1 \mathrm{GeV}$ (lower curves) [5 with our LO (dashed lines) and NLO (solid lines) fit results [8]

the H1 and ZEUS Collaborations [16] at HERA, and $\gamma \gamma$ data from the OPAL Collaboration [25] at LEP2. In hadroproduction and photoproduction, we set $\mu_{R}=\mu_{F}=\xi p_{T}$. As for the parton density functions (PDFs) of the proton, we employed set CTEQ5M provided by the CTEQ Collaboration [26], with $\Lambda \frac{(5)}{\mathrm{MS}}=226 \mathrm{MeV}$. As for the photon PDFs, we used the set by Aurenche, Fontannaz, and Guillet (AFG) [27]. In all cases, we found reasonable agreement between the experimental data and our NLO predictions as for both normalization and shape, as may be seen from Fig. 2. We conclude that our global analysis of inclusive charged-hadron production provides evidence that both the predicted scaling violations and the universality of the FFs are realized in nature.

\section{Inclusive $B$-Meson Production}

The QCD-improved parton model implemented in the $\overline{\mathrm{MS}}$ renormalization and factorization scheme and endowed with nonperturbative FFs, which proved itself so convincingly for light- [8, 8, 13] and $D^{* \pm}$-meson [10] inclusive production, also provides an ideal theoretical framework for a coherent 

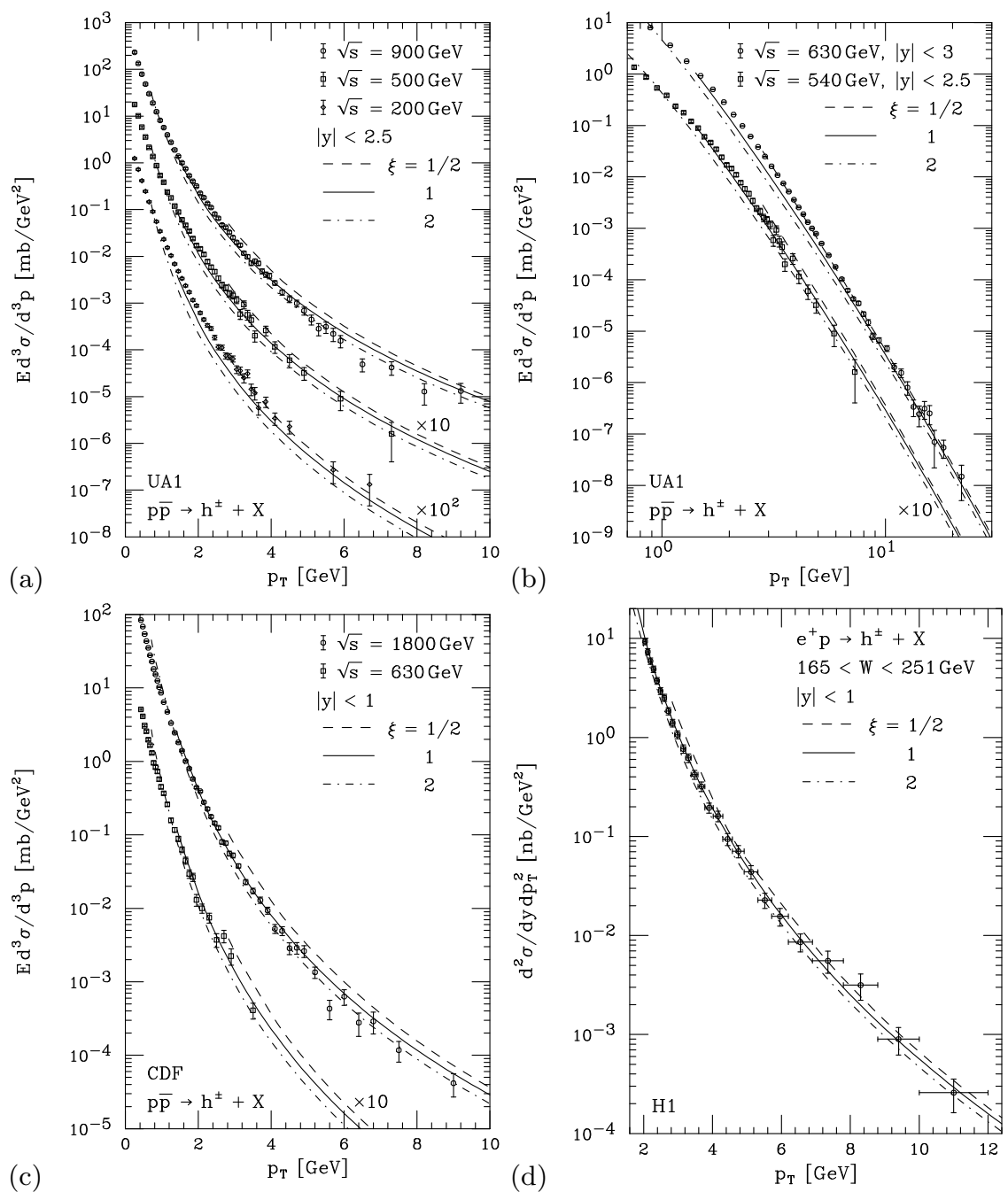

Fig. 2. Comparisons of $(\mathbf{a}, \mathbf{b}) \operatorname{S} p \bar{p} \mathrm{~S} \| 14$ and (c) Tevatron 15 data of $p \bar{p} \rightarrow h^{ \pm}+X$ and (d) HERA data of $\gamma p \rightarrow h^{ \pm}+X$ 16] with our NLO predictions [13]

global analysis of $B$-meson data [11], provided that $\mu \gg m_{b}$, where $\mu$ is the energy scale characteristic for the respective production process. Then, at LO (NLO), the dominant logarithmic terms, of the form $\alpha_{s}^{n, n+1} \ln ^{n}\left(\mu^{2} / m_{b}^{2}\right)$ with $n=1,2, \ldots$, are properly resummed to all orders by the AP evolution, while power terms of the form $\left(m_{b}^{2} / \mu^{2}\right)^{n}$ are negligibly small and can be safely neglected. The criterion $\mu \gg m_{b}$ is certainly satisfied for $e^{+} e^{-}$annihilation on the $Z$-boson resonance, and for hadroproduction of $B$ mesons with $p_{T} \gg m_{b}$. Furthermore, the universality of the FFs is guaranteed by 
the factorization theorem [28], which entitles us to transfer information on how $b$ quarks hadronize to $B$ mesons in a well-defined quantitative way from $e^{+} e^{-}$annihilation, where the measurements are usually most precise 19,29, to other kinds of experiments, such as hadroproduction [17,18].

In [11], the distribution in the scaled $B$-meson energy $x=2 E_{B} / \sqrt{s}$ measured by OPAL [19] at LEP1, which is compatible with the subsequent measurements by ALEPH at LEP1 and SLD at SLC [29], was fitted at LO and NLO using three different ansätze for the $b \rightarrow B \mathrm{FF}$ at the starting scale $\mu_{0}=2 m_{b}=10 \mathrm{GeV}$. The best fit was obtained for the ansatz by Peterson et al. (P) [30], with $\chi_{\mathrm{DF}}^{2}=0.67(0.27)$ at LO (NLO) [see Fig. 3(a)]. The $\varepsilon$ parameter was found to be $\varepsilon=0.0126$ (0.0198). We emphasize that the value of $\varepsilon$ carries no meaning by itself, but it depends on the underlying theory for the description of the fragmentation process $b \rightarrow B$, in particular, on the choice of the starting scale $\mu_{0}$, on whether the analysis is performed in $\mathrm{LO}$ or NLO, and on how the final-state collinear singularities are factorized in NLO. In Fig. 3(b), the $B^{+}$-meson $p_{T}$ distribution measured by CDF in Run 1A 17] and Run 1 18] is compared with our LO and NLO predictions evaluated using the CTEQ6 proton PDFs [31] and the BKK-P $B$-meson FFs [11 and choosing $\mu_{R, F}=\xi_{R, F} \times 2 m_{T}$, where $m_{T}=\sqrt{p_{T}^{2}+m_{b}^{2}}$. The theoretical uncertainty at NLO is estimated by independently varying $\xi_{R}$ and $\xi_{F}$ from 0.5 to 2 about the default value 1 . It only amounts to ${ }_{-27}^{+21} \%$ at $p_{T}=15 \mathrm{GeV}$, but steadily increases towards smaller values of $p_{T}$, reaching ${ }_{-45}^{+33} \%$ at $p_{T}=10 \mathrm{GeV}$. Variations in the proton PDFs and the ansatz for the $b \rightarrow B$ FF [11] only reach a few percent. We observe that the Run 1A data comfortably lies within the theoretical error band, while the full Run 1 data tends to be somewhat on the high side, especially in the upmost $p_{T}$ bin.

In the case of $\gamma \gamma \rightarrow D^{* \pm}+X$ at LEP2, the inclusion of finite- $m_{c}$ effects was found to reduce the cross section by approximately $20 \%(10 \%)$ at $p_{T}=2 m_{c}$ $\left(3 m_{c}\right)$ [32], i.e., their magnitude is roughly $m_{c}^{2} / p_{T}^{2}$, as naïvely expected. By analogy, one expects the finite- $m_{b}$ terms neglected in [1] to have a moderate size, of order $20 \%(10 \%)$ at $p_{T}=10 \mathrm{GeV}(15 \mathrm{GeV})$. This is considerably smaller than the scale uncertainty and appears insignificant compared to the excess of the CDF data [17.18] over the traditional NLO analysis [33] in a scheme with $n_{f}=4$ active quark flavours, where the $b$ quark is treated in the on-mass-shell renormalization scheme. In this massive scheme, there are no collinear singularities associated with the outgoing $b$-quark lines that need to be subtracted and absorbed into the FFs. In fact, in this scheme there is no conceptual necessity for FFs at all, but they are nevertheless introduced in an ad-hoc fashion in an attempt to match the $B$-meson data. However, in the absence of a subtraction procedure, there is also no factorization theorem in operation to guarantee the universality of the FFs [28]. By the same token, such FFs are not subject to AP evolution. Thus, there is no theoretical justification to expect, e.g., that a single value of the Peterson $\varepsilon$ parameter should be appropriate for different types of experiment or at different energy 


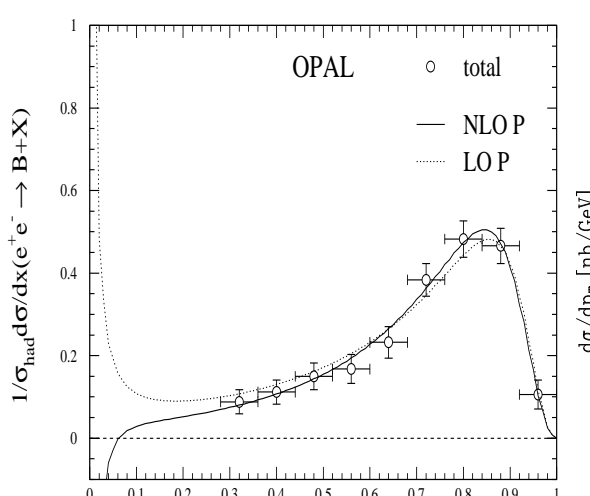

(a)

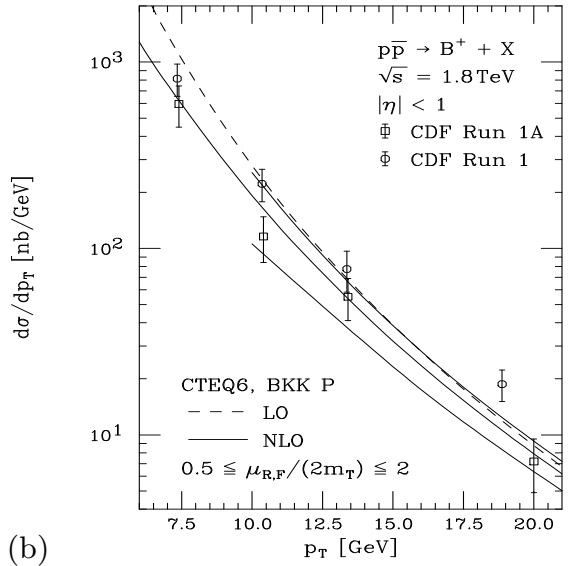

Fig. 3. (a) Our LO and NLO fits [11] to OPAL data of $e^{+} e^{-} \rightarrow B+X[19$. (b) Comparison of CDF data of $p \bar{p} \rightarrow B+X$ [17, 18] with our LO and NLO predictions 11

scales in the same type of experiment. In other words, the feasibility of global data analyses is questionable in this scheme. Moreover, this scheme breaks down for $p_{T} \gg m_{b}$ because of would-be collinear singularities of the form $\alpha_{s} \ln \left(p_{T}^{2} / m_{b}^{2}\right)$, which are not resummed.

The attempt to split the $B$-meson FFs into a so-called perturbative FF $(\mathrm{PFF})$ and a nonperturbative remainder is interesting in its own right. However, detailed analysis for $D^{* \pm}$-meson FFs 34 revealed that such a procedure leads to deficient results in practical applications. On the one hand, at NLO, the cross section $d \sigma / d x$ of $e^{+} e^{-}$annihilation becomes negative in the upper $x$ range, at $x \gtrsim 0.9$ [see Fig. 1(a)], where the data is very precise, so that a low-quality fit is obtained unless this $x$ range is excluded by hand [34,35]. On the other hand, the LO and NLO predictions for other types of processes, such as photoproduction in $e p$ scattering [see Fig. A(b)], significantly differ [34, which implies that the perturbative stability is insufficient.

The idea 11] of performing a coherent analysis of LEP1 and Tevatron data of inclusive $B$-meson production was recently revived using an unconventional scheme named FONLL, in which the traditional result in the massive scheme [33 and a suitably subtracted result in a massless scheme with PFFs are linearly combined [36]. The degree of arbitrariness in this procedure may be assessed by noticing that the massless-scheme term is weighted with an ad-hoc coefficient function of the form $p_{T}^{2} /\left(p_{T}^{2}+25 m_{b}^{2}\right)$ so as to effectuate its suppression in the low- $p_{T}$ range and that this term is evaluated at $p_{T}^{\prime}=\sqrt{p_{T}^{2}+m_{b}^{2}}$ while the massive-scheme term is evaluated at $p_{T}$. Since the FONLL scheme interpolates between the massive scheme and the massless scheme with PFFs, it inherits the weaknesses of both schemes detailed above. 

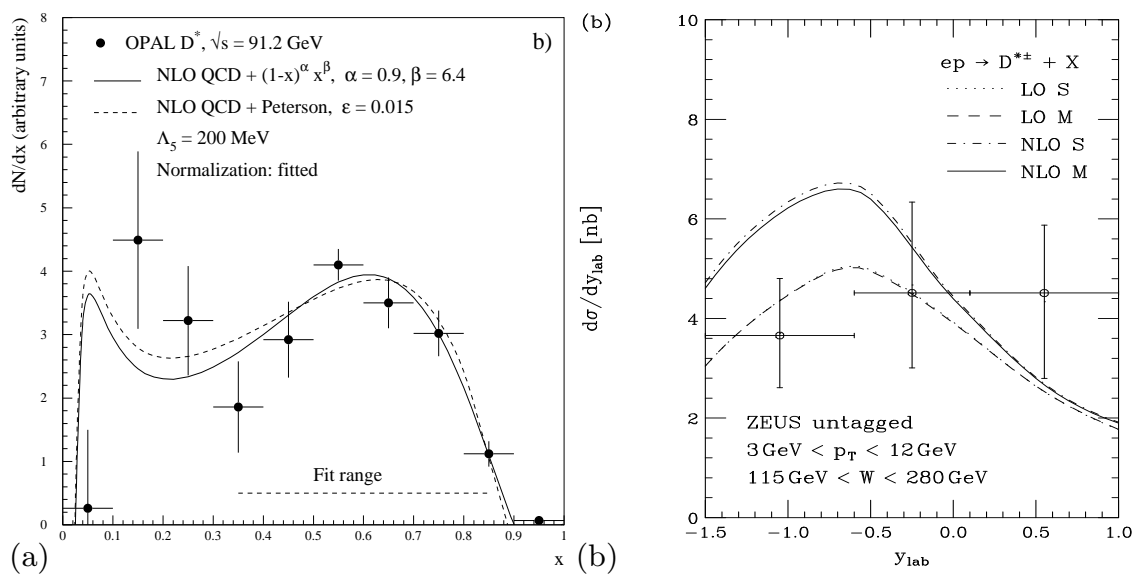

Fig. 4. (a) NLO fit 35 to OPAL data of $e^{+} e^{-} \rightarrow D^{* \pm}+X$ using PFFs. (b) Comparison of ZEUS data of $e p \rightarrow D^{* \pm}+X$ in photoproduction with our LO and NLO predictions implemented with PFFs 34

In particular, the negativity of the NLO cross section of $e^{+} e^{-} \rightarrow B+X$ in the upper $x$ range forces one to exclude the data points located there from the fit. In [36], this is achieved by resorting to what is called there the moments method, i.e., the large- $x$ region is manually faded out by selecting one particular low moment of the $b \rightarrow B \mathrm{FF}$, namely the one corresponding to the average $x$ value, thereby leaving the residual information encoded in the data unused.

\section{Conclusions}

We reviewed recent LO and NLO analyses of $\pi^{ \pm}, K^{ \pm}$, and $p / \bar{p}$ FFs [8], which also yielded new values for $\alpha_{s}^{(5)}\left(M_{Z}\right)$ [12. Although these FFs are genuinely nonperturbative objects, they possess two important properties that follow from perturbative considerations within the QCD-improved parton model and are amenable to experimental tests, namely scaling violations and universality. The scaling violations were tested [8,133 by making comparisons with data of $e^{+} e^{-}$annihilation at $\mathrm{CM}$ energies below [20] and above [24] those pertaining to the data that entered the fits. The universality property was checked [13] by performing a global study of high-energy data on hadroproduction in $p \bar{p}$ collisions [14,15] and on photoproduction in $e^{ \pm} p$ [16] and $e^{+} e^{-}$25] collisions. Our NLO FFs [8] agree with other up-to-date sets [37 within the present experimental errors 13 .

High-energy data on hadroproduction particularly probes the FFs in the large- $x$ region, is especially sensitive to the gluon FFs, and, therefore, carries valuable information complementary to that provided by $e^{+} e^{-}$data. This 
information, apart from being interesting in its own right, is indispensible in order to reliably predict the $\pi^{0}$ background for the $H \rightarrow \gamma \gamma$ signal of the intermediate-mass Higgs boson at the Tevatron and the LHC. It would thus be highly desirable if the experiments at the Tevatron made an effort to update their analyses, which date back to 1988 [15].

Adopting a similar theoretical framework for $B$-meson production, we demonstrated [11] that the notorious excess of the Tevatron data 17, 18, over existing theoretical calculations can be ascribed, at sufficiently large values of $p_{T}$, to nonperturbative fragmentation effects inappropriately taken into account previously. In combination with the factorization formalism of nonrelativistic QCD [38], this framework also leads to a successful description [39] of inclusive $J / \psi$ and $\psi^{\prime}$ production from $B$-meson decay [40]. A rigorous procedure to implement the finite- $m_{b}$ terms in an NLO framework where terms of the form $\alpha_{s}^{n+1} \ln ^{n}\left(\mu^{2} / m_{b}^{2}\right)$ are resummed by AP evolution and the univer-

sality of the FFs is guaranteed by the factorization theorem [28] is to directly subtract the would-be collinear singularities of the form $\alpha_{s} \ln \left(p_{T}^{2} / m_{b}^{2}\right)$ in the massive-scheme result in a way that conforms with the massless scheme [32] (see also 41).

\section{Acknowledgements}

The author is grateful to J. Binnewies, G. Kramer, and B. Pötter for their collaboration on the work presented here. His research is supported in part by DFG Grant No. KN 365/1-1 and BMBF Grant No. 05 HT1GUA/4.

\section{References}

1. V.N. Gribov, L.N. Lipatov: Yad. Fiz. 15, 781 (1972) [Sov. J. Nucl. Phys. 15, 438 (1972)]; G. Altarelli, G. Parisi: Nucl. Phys. B 126, 298 (1977); Yu.L. Dokshitser: Zh. Eksp. Teor. Fiz. 73, 1216 (1977) [Sov. Phys. JETP 46, 641 (1977)]

2. ALEPH Collaboration, D. Buskulic et al.: Phys. Lett. B 357, 487 (1995); 364, 247(E) (1995)

3. DELPHI Collaboration, P. Abreu et al.: Phys. Lett. B 398, 194 (1997); OPAL Collaboration, K. Ackerstaff et al.: Eur. Phys. J. C 7, 369 (1999)

4. ALEPH Collaboration, D. Buskulic et al.: Z. Phys. C 66, 355 (1995); DELPHI Collaboration, P. Abreu et al.: Eur. Phys. J. C 5, 585 (1998); SLD Collaboration, K. Abe et al.: Phys. Rev. D 59, 052001 (1999)

5. ALEPH Collaboration, R. Barate et al.: Eur. Phys. J. C 17, 1 (2000); OPAL Collaboration, G. Abbiendi et al.: Eur. Phys. J. C 11, 217 (1999)

6. DELPHI Collaboration, P. Abreu et al.: Eur. Phys. J. C 13, 573 (2000)

7. J. Binnewies, B.A. Kniehl, G. Kramer: Z. Phys. C 65, 471 (1995); Phys. Rev. D 52, 4947 (1995)

8. B.A. Kniehl, G. Kramer, B. Pötter: Nucl. Phys. B 582, 514 (2000)

9. TPC/Two-Gamma Collaboration, H. Aihara et al.: LBL Report No. LBL-23737 and UC-34D, March 1988 (unpublished); Phys. Rev. Lett. 61, 1263 (1988) 
10. J. Binnewies, B.A. Kniehl, G. Kramer: Phys. Rev. D 58, 014014 (1998)

11. J. Binnewies, B.A. Kniehl, G. Kramer: Phys. Rev. D 58, 034016 (1998)

12. B.A. Kniehl, G. Kramer, B. Pötter: Phys. Rev. Lett. 85, 5288 (2000)

13. B.A. Kniehl, G. Kramer, B. Pötter: Nucl. Phys. B 597, 337 (2001)

14. UA1 Collaboration, G. Arnison et al.: Phys. Lett. 118 B, 167 (1982); UA2 Collaboration, M. Banner et al.: Phys. Lett. 122 B, 322 (1983); Z. Phys. C 27, 329 (1985); UA1 Collaboration, C. Albajar et al.: Nucl. Phys. B 335, 261 (1990); UA1 Collaboration, G. Bocquet et al.: Phys. Lett. B 366, 434 (1996)

15. CDF Collaboration, F. Abe et al.: Phys. Rev. Lett. 61, 1819 (1988)

16. ZEUS Collaboration, M. Derrick et al.: Z. Phys. C 67, 227 (1995); H1 Collaboration, C. Adloff et al.: Eur. Phys. J. C 10, 363 (1999)

17. CDF Collaboration, F. Abe et al.: Phys. Rev. Lett. 75, 1451 (1995)

18. CDF Collaboration, D. Acosta et al.: Phys. Rev. D 65, 052005 (2002)

19. OPAL Collaboration, G. Alexander et al.: Phys. Lett. B354, 93 (1995)

20. DASP Collaboration, R. Brandelik et al.: Nucl. Phys. B 148, 189 (1979); TASSO Collaboration, M. Althoff et al.: Z. Phys. C 17, 5 (1983); TASSO Collaboration, W. Braunschweig et al.: Z. Phys. C 42, 189 (1989); ARGUS Collaboration, H. Albrecht et al.: Z. Phys. C 44, 547 (1989)

21. OPAL Collaboration, R. Akers et al.: Z. Phys. C 68203 (1995); DELPHI Collaboration, P. Abreu et al.: Eur. Phys. J. C 6, 19 (1999)

22. Particle Data Group, K. Hagiwara et al.: Phys. Rev. D 66, 010001 (2002)

23. K.G. Chetyrkin, B.A. Kniehl, M. Steinhauser: Phys. Rev. Lett. 79, 2184 (1997)

24. OPAL Collaboration, G. Alexander et al.: Z. Phys. C 72, 191 (1996); OPAL Collaboration, K. Ackerstaff et al.: Z. Phys. C 75, 193 (1997); DELPHI Collaboration, P. Abreu et al.: Phys. Lett. B 459, 397 (1999); OPAL Collaboration, G. Abbiendi et al.: Eur. Phys. J. C 16, 185 (2000)

25. OPAL Collaboration, K. Ackerstaff et al.: Eur. Phys. J. C 6, 253 (1999)

26. CTEQ Collaboration, H.L. Lai et al.: Eur. Phys. J. C 12, 375 (2000)

27. P. Aurenche, J.-P. Guillet, M. Fontannaz: Z. Phys. C 64, 621 (1994)

28. J.C. Collins: Phys. Rev. D 58, 094002 (1998); private communication

29. ALEPH Collaboration, A. Heister et al.: Phys. Lett. B 512, 30 (2001); SLD Collaboration, K. Abe et al.: Phys. Rev. D 65, 092006 (2002)

30. C. Peterson, D. Schlatter, I. Schmitt, P.M. Zerwas: Phys. Rev. D 27, 105 (1983)

31. CTEQ Collaboration, J. Pumplin et al.: JHEP 0207, 012 (2002)

32. G. Kramer, H. Spiesberger: Eur. Phys. J. C 22, 289 (2001)

33. P. Nason, S. Dawson, R.K. Ellis: Nucl. Phys. B 327, 49 (1989); 335, 260(E) (1990); W. Beenakker, H. Kuijf, W.L. van Neerven, J. Smith: Phys. Rev. D 40, $54(1989)$

34. J. Binnewies, B.A. Kniehl, G. Kramer: Z. Phys. C 76, 677 (1997)

35. M. Cacciari, M. Greco: Phys. Rev. D 55, 7134 (1997)

36. M. Cacciari, P. Nason: Phys. Rev. Lett. 89, 122003 (2002)

37. S. Kretzer: Phys. Rev. D 62, 054001 (2000); L. Bourhis, M. Fontannaz, J.P. Guillet, M. Werlen: Eur. Phys. J. C 19, 89 (2001)

38. G.T. Bodwin, E. Braaten, G.P. Lepage: Phys. Rev. D 51, 1125 (1995); 55, 5853(E) (1997)

39. B.A. Kniehl, G. Kramer: Phys. Rev. D 60, 014006 (1999)

40. CDF Collaboration, F. Abe et al.: Phys. Rev. Lett. 79, 572 (1997); D0 Collaboration, B. Abbott et al.: Phys. Rev. Lett. 82, 35 (1999)

41. F.I. Olness, R.J. Scalise, W.-K. Tung, Phys. Rev. D 59, 014506 (1999) 